\title{
Does Mixed Spices Affect Serum Insulin and Adiponectin Concentration in male Sprague Dawley Rats?
}

\author{
Muhammad Muzaffar Ali Khan Khattak ${ }^{1,2,3, *}$, Ibrahim Abu Bakar ${ }^{1}$, Nik Mazlan Mamat ${ }^{1}$, \\ Wan Azdie Mohd Abu -Bakar ${ }^{1}$, Nor Azwani Mohd-Shukri ${ }^{1}$ \\ ${ }^{1}$ Department of Nutrition Sciences, Kulliyyah of Allied Health Sciences, International Islamic University, Malaysia, Jalan Sultan \\ Ahmed Shah, Bandar Indera Mahkota Kuantan, 25200, Pahang Darul Makmur, MALAYSIA \\ ${ }^{2}$ Non Communicable Diseases Research Unit, Kulliyyah of Medicine , International Islamic University Malaysia (IIUM) Jalan Sultan \\ Ahmad Shah, Bandar Indera Mahkota, 25200 Kuantan, Pahang Darul Makmur, Malaysia \\ ${ }^{3}$ International Institute for Halal Research and Training (INHART), E5 2-2, Level 2, Block E5, Kulliyyah of Engineering, International \\ Islamic University Malaysia, P.O. Box 10 Kuala Lumpur, MALAYSIA \\ *Corresponding author: mkbiol@yahoo.com
}

Received September 09, 2014; Revised September 17, 2014; Accepted September 23, 2014

\begin{abstract}
Spices are used in food around the world for enhancing the flavour/aroma of dietary preparations. Apart from this aspect spices are also used in herbal medicines practice/treatment. However, the effect of mixed spices namely cinnamon (Cinnamomum zeylanicum), cloves (Syzygium aromaticum or Eugenia caryophyllata), turmeric (Curcuma longa), and bay leaves also known as curry leaf (Murraya koenigii) on serum glucose, insulin and adiponectin protein concentration in rats induced hyperglycemia with streptozotocin (STZ) have not been studied. Sprague Dawley rats aged three months were injected with STZ (40 mg/kg body weight) in the abdomen in order to induce hyperglycemia. The rats were acclimatized with diets prior allocation to the doses of mixed spices. After having developed hyperglycemia, the rats were divided into four groups i.e. 0 (control), one, two and three g per day. The aforementioned spices were ground and mixed in equal ratios and added to stock diets 0, 1, 2 and 3 gram of the mixed spices in the feed to be fed to rats daily. The total duration of the feeding was 40 days and followed by 20 days after effect of the spices. Blood was collected on the day 0, 21, 31, 41 from the tail and on the 61st day from heart puncture of the rats. The blood samples were immediately processed for serum separation after day 61 collection and stored for later analysis at -70 C. The serum was analyzed for adiponectin protein, insulin and glucose. The statistical analysis of the results indicated that serum adiponectin protein and insulin concentration were significantly $(\mathrm{P}<0.05)$ higher whereas serum triglycerides concentration was significantly $(\mathrm{P}<0.05)$ lower in three gram fed spices compared to control 1 and 2 gram spices fed groups. This study indicates that spices provide control over hyperglycemia through increased adiponectin and insulin in circulating blood. Therefore, these spices combination may be used for the control of hyperglycaemia in type 2 diabetic patients.
\end{abstract}

Keywords: rats, mixed spices, glucose, adiponectin, insulin

Cite This Article: Muhammad Muzaffar Ali Khan Khattak, Ibrahim Abu Bakar, Nik Mazlan Mamat, Wan Azdie Mohd Abu-Bakar, and Nor Azwani Mohd-Shukri,"Does Mixed Spices Affect Serum Insulin and Adiponectin Concentration in male Sprague Dawley Rats?” Journal of Food and Nutrition Research, vol. 2, no. 10 (2014): 681-685. doi: 10.12691/jfnr-2-10-5.

\section{Background}

Diabetes is a group of metabolic diseases characterized by persistent higher concentration of glucose (hyperglycemia) resulting from defects in insulin secretion, insulin action, or both which include all types of diabetes. The type 2 diabetes specifically refers to less insulin secretion from the beta cells of pancreas and defect in insulin action on the glucose entry to the cells. In general the terms hyperglycemia and hypoglycemia are used for higher or lower concentration of glucose in circulating blood. According to the World Health Organization (WHO), Type 2 diabetes affects more than 171000,000 people around the world and the incidence is expected to be $366,000,000$ by the year 2030 . The number reported for the Peninsular Malaysia is 942,000 and this will be increased to $2,479,000$ by the year 2030 [1], which is an alarming situation around the world including Peninsular Malaysia. Type 2 diabetes and obesity are generally considered to be multifactorial with an increased risk of mortality and morbidity [2]. The causes and cure of diabetes are not entirely clear but diet certainly plays an important role. Hyperglycemia may be controlled through various means i.e. diet, physical activity and pharmacological manipulations, while manipulations often increase the insulin secretions [3]. Dietary manipulations are the most desirable one in which there is least burden on the pancreatic functional ß-cells. Spices which are added to the preparation of diets for taste and aroma have been shown to be effective in the control of 
hyperglycemia both in animals and human studies. It has been reported that these spices have insulin potentiatingfactor and enhanced chromium contents; it has been shown previously that components in cinnamon (a spice) improve insulin activity through an unidentified factor present in it [4] later characterized as methyl-hydroxychalcone polymer [5]. In human study, the effects of cinnamon on blood sugar and lipid profile are remarkable in type 2 diabetic individuals [6]. In animal model it has been shown that adiponectin (an adipokine secreted by adipose tissues act like hormone and is considered useful for energy utilization) improves insulin (pancreatic hormone secreted by the $\beta$-cells of pancreas which helps in glucose entry to the cells) sensitivity in insulin resistant animals. Low plasma adiponectin levels have been shown to be a marker of developing type 2 diabetes in humans. Adiponectin is also inversely associated with other traditional cardiovascular risk factors, such as blood pressure, total and low-density lipoprotein (LDL) cholesterol and triglyceride concentrations and is positively related to high-density lipoprotein (HDL) cholesterol concentrations. Furthermore, recent studies suggested that it may have anti-atherogenic and antiinflammatory properties. Similarly, in humans, plasma adiponectin concentration negatively correlates with basal metabolic index (BMI), fasting insulin concentration, and serum triglyceride $[7,8]$. Weight reduction achieved by allow-calorie diet also increases plasma adiponectin concentration in both non-diabetic and diabetic patients [9].

Spices are used in food recipes around the world with a view to enhance the flavor and aroma of dietary preparations. Apart from this aspect, spices are also used in herbal medicines practice/treatments. Therefore, spices have been studied extensively in relation to their effectiveness in the prevention/control of certain disease conditions. Numerous studies have shown that spices consumed as a part of food provides best control over diabetes (hyperglycemia). If, this is the case with the adiponectin in relation to the stated parameters then the mixed spices must have enhancing effect on the adiponectin protein in the rat's model. This project was designed to study the effect of the mixed spices (i.e. cinnamon or Cinnamomum zeylanicum, cloves or Syzygium aromaticum sometimes called as Eugenia caryophyllata, turmeric or Curcuma longa, and bay leaves also known as curry leaf or Murraya koenigii on adiponectin protein in blood, blood glucose, insulin and lipid profile in rats model.

\section{Methods}

Forty Eight Sprague Dawley rats were obtained from the Kulliyyah of Science, International Islamic University Malaysia, Kuantan. The rats of similar age were used and housed one per cage and were kept in 12- Hours light and dark under standard lighting conditions. In this experiment, four spices i.e. cinnamon (Cinnamomum zeylanicum), cloves (Syzygium aromaticum), turmeric (Curcuma longa) and bay leaves (Murraya koenigii) were ground and mixed in the ratio of 25:25:25:25 grams/100 grams of mixed spices. The rats were fed in three different doses 1, 2 and 3 gram per day in the feed. One basal diet (as indicated in Table 1 Gold-Coin Brand, Special Rations for Mouse) was prepared and manipulated for the test diets just by adding/adjusting for $0,1,2$ and 3 gram of the mixed spices and fed 20 gram of feed per day (The dose was calculated for rats based on the assumption of $60 \mathrm{~kg}$ man). Thus there were four diets namely Basal-diet, Test-diet1, Test-diet2 and Test-diet3 having calculated doses of $0(0)$, 1 (0.025 g), 2 (0.050 g), \& 3 grams (0.075 g) of mixed spices/day.

Table 1. Composition of the diet $(\mathrm{g} / \mathrm{kg})$

\begin{tabular}{ccccc}
\hline Ingredients (\%) & 0 g Mixed Spice (Basal) & 1g Mixed Spice (test-diet1) & 2g Mixed Spice (Test-diet2) & 3g Mixed Spice (Test-diet3) \\
\hline Protein & 21.00 & 21.00 & 21.00 & 21.00 \\
Carbohydrates & 49.00 & 49.00 & 49.00 & 5.00 \\
Fire & 5.00 & 5.00 & 3.00 & 5.00 \\
Fat & 3.00 & 3.00 & 13.00 & 3.00 \\
Moisture & 13.00 & 13.00 & 8.00 & 13.00 \\
Ash & 8.00 & 8.00 & 1.00 & 8.00 \\
Additives & 1.00 & 1.00 & 100.00 & 1.00 \\
\hline Total & 100.00 & 100.00 & 100.00 \\
\hline
\end{tabular}

Gold-Coin Brand, Special Rations, Mouse Pellets (702P)

The rats were allowed to acclimatize for period of one week on the basal diet and later randomly allocated six rats to each of the diets. The rats were induced hyperglycemia by using Streptozotocin (STZ, procured from MERCK SdnBhd, Level 3, Menara Sunway Annexe, Jalan Lagoon Timur, Bandar Sunway, 46150 Petaling Jaya, Selangore Darul Ehsan, Malaysia) during the acclimatization period. The STZ was induced intraperitoneally to the rats at a dose of $40 \mathrm{mgs} / \mathrm{kg}$ body (calculated according the body weight of the rat). The rats were assessed for the development of hyperglycemia during the acclimatization period. The tailed blood glucose concentration higher than 7.0 mmoles/l (126 $\mathrm{mgs} / \mathrm{dl}$ ) in fasting states was considered as hyperglycemic according [10] WHO, 2006 recommendation. After having developed hyperglycemia the rats were put on experimental feed for duration of 20, 30, 40 and 60 days. In the days 1 to 40 the rats received the aforementioned doses of spices and in the last 20 days there were no supplementation of the diets with spices. Each dose group was having six rats per group. Thus there were 24 rats in total in each stage of the experiment.The rat's blood was 
collected on the day $0,21,31,41$ from the tail and on the $61^{\text {st }}$ day from heart puncture of the rats. The blood samples were immediately processed for serum separation and stored for later analysis at $-70 \mathrm{C}$. Blood was used for the analysis of glucose, insulin and adiponectin protein. Serum glucose was measured by kit methods from Bayer Health Care (Bayer Diagnostics Europe Limited Chapel Lane Swords, Co. Dublin, Ireland, UK) on a clinical analyzer. Serum insulin concentration was estimated by using competitive colorimetric enzyme-linked immunosorbent assay (ELISA) kit method from SPIBIO Berten Medical Device and Equipment for Life Science Laboratories and Environment Surveillance, Parcd' Activités du Pas du Lac 10 bis avenue ampère F-78180 Montigny le Bretonneux FRANCE (Paris outskirts). Plasma adiponectin protein concentration was estimated by using competitive calorimetric enzyme-linked immunosorbent assay (ELISA) kit known as the B-bridge adiponectin ELISA kit method by Mercodia AB, Sylveniusgatan, A, SE-75450 Uppsala, Sweden. The experimental protocol of the study was approved from the university.
The results were statistically analyzed and were expressed as the means \pm SD. The statistical significance/difference was determined using the Minitab statistical software. The significance level was set at 95 percent $(P<0.05)$ confidence interval.

\section{Results}

The consumption of feed is shown in the Table 2 . Generally, the dietary intakes were $100 \%$ among the four groups which is of great importance for the comparison of the effect of diets between the dietary groups and within the animals. Apparently, the performance of rats and the general wellbeing in the groups could be ranked in the order of best to poor like the Test-diet3>Test-diet2>Testdiet1>Basal diet as shown in the in the Table 2. This is evident in the form of feed intakes and physical appearances of the rats in the different diet groups the mean refusal was minimal at duration $1,2,3$ and 4 . The mean refusal for the different duration did not varied significantly as indicated in the Table 2.

Table 2. Dietary Intakes and Refusal of the Rats

\begin{tabular}{|c|c|c|c|c|}
\hline & Basal (0g) & Test-diet1 (1g) & Test-diet2 (2g) & Test-diet3 (3g) \\
\hline Total Feed Intake (g) & $1110 \pm 37$ & $1109 \pm 89$ & $1133 \pm 95$ & $1129 \pm 60$ \\
\hline Feed Intake (g/Day) & $19 \pm 0.61$ & $19 \pm 1.48$ & $19 \pm 1.58$ & $192 \pm 1.01$ \\
\hline Percent Intake/Day & 93 & 92 & 94 & 94 \\
\hline Total Feed Refusal (g) & $90 \pm 37$ & $91 \pm 81$ & $67 \pm 95$ & $71 \pm 61$ \\
\hline Feed Refusal (g/Day) & $1.45 \pm 0.61$ & $1.52 \pm 1.48$ & $1.12 \pm 1.58$ & $1.18 \pm 1.01$ \\
\hline Percent Refusal/Day & 7.25 & 7.60 & 5.5 & 5.9 \\
\hline
\end{tabular}

The mean body weight of the rats was reduced after the development of hyperglycemia however it was not different for the groups. The general performance/wellbeing was best in the three gram fed rats (Figure 1).
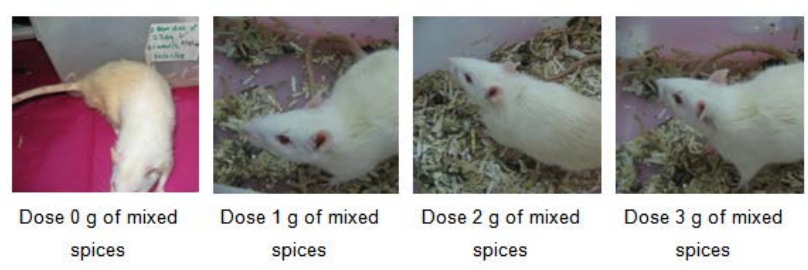

Figure 1. Dose effect of mixed spices on the general well being of rats

\subsection{Effect of Mixed Spices on Blood and Serum Glucose Concentration}

Blood and serum glucose concentration was significantly lower $(\mathrm{P}<0.05)$ for the three grams mixed spices fed group compared to control, one gram and two grams fed spices measured in whole blood and in serum (Figure 2).

The after effect was continued in three gram fed rats until the day $61^{\text {st }}$ whereas no effect of other doses was observed. When assessed the percent reduction of glucose concentration in the serum was higher in the 3 gram fed spices group as indicated in Figure 4.

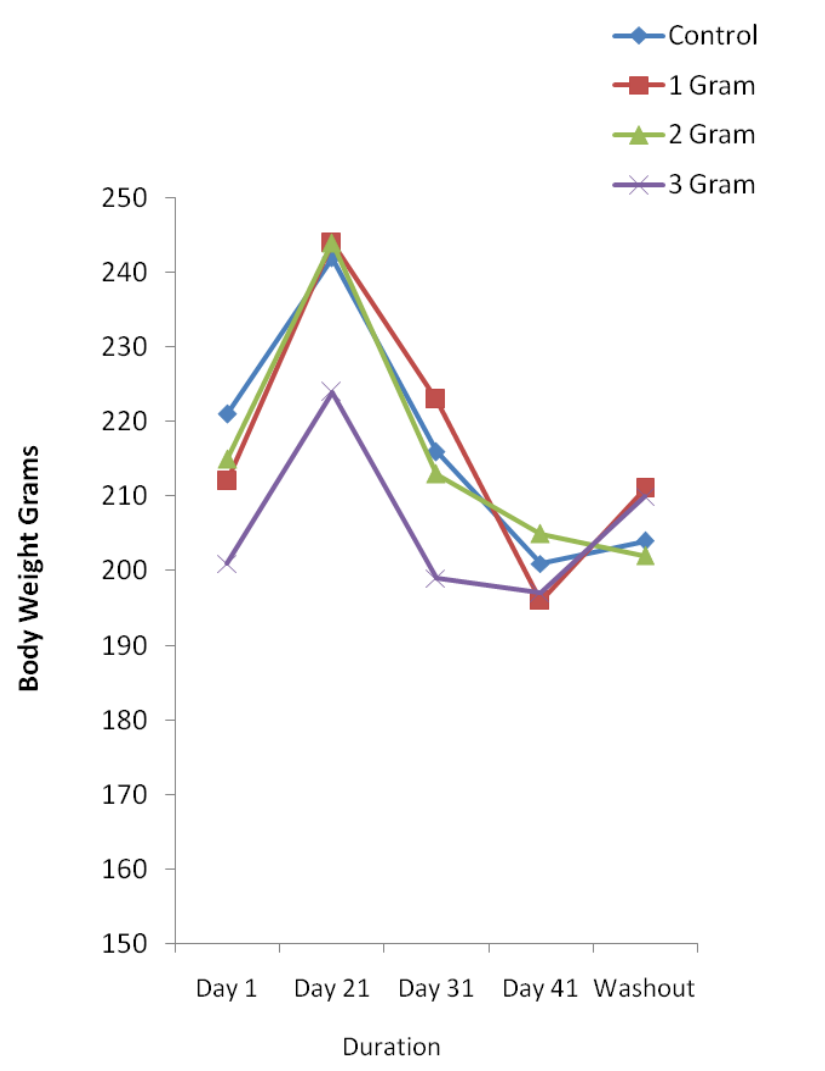

Figure 2. Effect of different doses on the body weight rats 


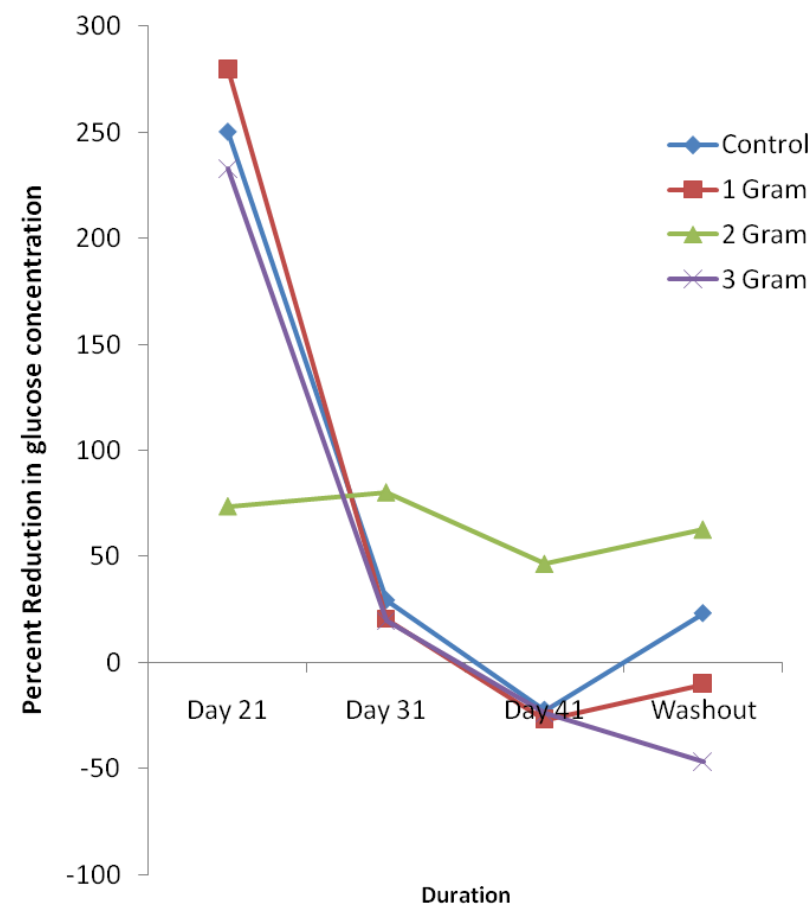

Figure 3. Effect of diets on hyperglycemia in STZ induced hyperglycemic rats

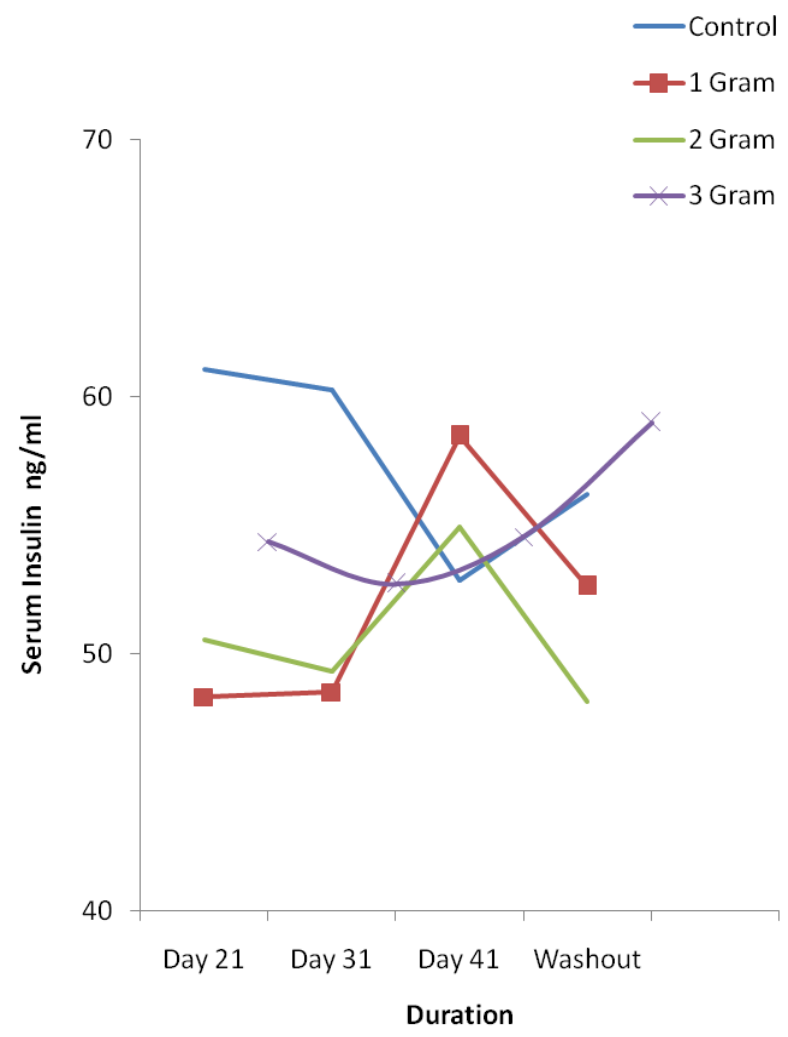

Figure 4. Effect of Mixed Spices on Serum Insulin concentration

The serum insulin concentration was significantly higher on day $61^{\text {st }}$ compared to the rest of the three groups until the feeding period but the effect continue to rise after feeding period in the three gram fed group. Furthermore, there was steady increase in the three gram fed group (Figure 4). Serum adiponectin concentration was assayed and it was significantly higher for the day $41^{\text {st }}$ and $61^{\text {st }}$ compared to the day one, $31^{\text {st }}$ in three gram fed group. The effect on the adiponectin seems to be persistent after the feeding period (Figure 5).

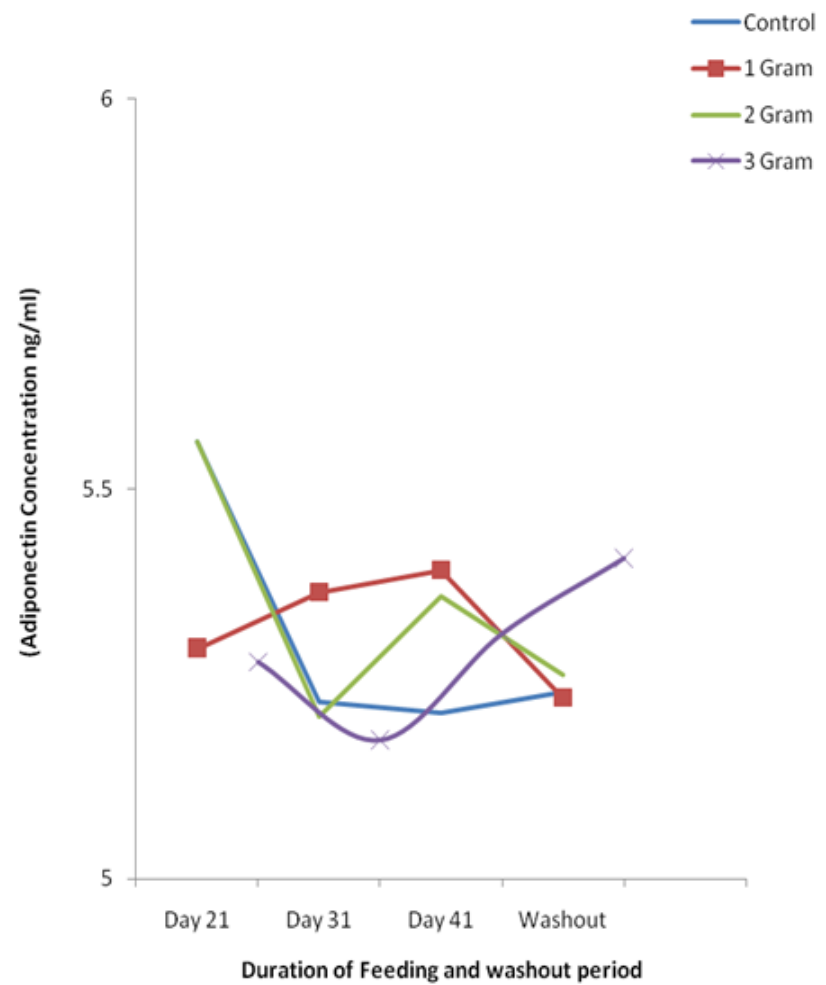

Figure 5. Effect of mixed spices on serum adiponectin concentration (ng/ml)

\section{Discussion}

The effect of spices has been studied on the different aspects of diabetes and hyperlipidaemia. The present approach differs from the earlier in the sense that spices used in this study have not been used in the ratio 25\% (mixed spices). The effect of mixed spices was encouraging on serum glucose and adiponectin concentration on the STZ induced diabetic rats. These observations may be of clinical significance to the nutritionist, dietician and of course to clinicians involved in this area of research and practice. In earlier study conducted by our group have already established that three gram of cinnamon feeding to type 2 diabetes mellitus patients is effective in controlling blood glucose and LDL$c$ and have significant effect both on serum glucose and serum LDL-c concentration $[6,11]$. However, the effect of mixed spices remained unclear; this study shows three grams of mixed spices have similar effect in the STZ induced diabetes rats. In the current literature, it is widely advocated that adiponectin (the product of the fat cell secretion) is negatively associated with energy, blood glucose and LDL-concentration. This particular action of mixed spice on adiponectin and insulin has never been addressed. In the present study, mixed spices provided better control over hyperglycemia and this action was accompanied with physically healthier looking. The difference was apparent among the four groups as indicated in the Figure 1. In diabetes both human and animal subjects with increased thirst (polydipsia), excessive hunger (polyphagia), excessive weight loss (emaciation), extreme fatigue or lack of energy, excessive urine (polyuria) and blurred vision are common signs, \& symptoms etc [12]. These signs were evident in the 
control group with a significant weight loss as indicated in the Figure 2.

This study also shows that the insulin concentration was higher and remained higher after the day 40 of the feeding of mixed spices in the three gram fed group. It has been reported that the dietary manipulations often increases the insulin secretions and this happen in this case [3]. There are various theories and postulations regarding the action of spices on the blood glucose, lipids and secretion of insulin from the pancreas including the insulin potentiating factor. In animal model, it has been shown that adiponectin increase insulin sensitivity in insulin resistance. Similarly, Low plasma adiponectin concentrations have been used as a marker of developing type II diabetes mellitus in humans subjects. Adiponectin is also inversely associated with other traditional cardiovascular risk factors, such as blood pressure, total and low-density lipoprotein (LDL) cholesterol and triglyceride concentrations and is positively related to high-density lipoprotein (HDL) cholesterol concentrations. Furthermore, recent studies suggest that adiponectin may have anti-atherogenic and anti-inflammatory properties. Similarly, in humans, plasma adiponectin concentration is negatively correlated with BMI, fasting insulin concentration, and serum triglyceride $[7,8]$. Weight reduction achieved by a low-calorie diet also increases plasma adiponectin concentration in both non-diabetic and diabetic patients [9]. The suggestions provided in these articles are of significant importance to this observation of spice being negatively associated with glucose and positively associated with insulin and adiponectin concentration in the serum of STZ-induced diabetic rate. Our recent studies on mixed spices, mixed herbs and a mixtures of spices \& herbs in thirty days clinical trial in type II diabetic patients have shown significant effect in blood glucose concentration with improving trend in lipid profile $[13,14,15]$.

\section{Conclusions}

If, these observations are observed consistently that the mixed spices have the ability to reduce glucose and increase the insulin \& adiponectin then this property may be used as dietary therapy of hyperglycemia and obesity in the form of drinks or can be incorporated to food items. Therefore, it is suggested further studies may be carried out in the type II diabetic, metabolic syndrome and obese patients.

\section{Acknowledgements}

The authors are thankful to Research Mamangement Centre, International Islamic university Malaysia (IIUM) for funding this project (Project No. IFRG 0701-36).

\section{References}

[1] World Health Organization. Publication of the World Health Organization. WHO Press, 20 Avenue Appia, 1211 Geneva 27, Switzerland. 2005.

[2] Rosenbaum M, Leibel RL, Hirsch J. (1997). Obesity. New England Jornal of Medicines, 337, 396-407.

[3] Kaiser N, Leibowitz G, Nesher R. (2003). Glucotoxicity and betacell failure in type 2 diabetes mellitus. Journal of Pediatric Endocrinology and Metabolism, 16, 5-22.

[4] Khan A, Bryden MM, Anderson RA, Polansky MM. (1990). Insulin potentiating factor and chromium content of selected food and spices. Biological Trace Element Research, 24, 183-188.

[5] Anderson RA, Broadhurst CL, Polansky MM., Schmidt WF, Khan A, Schoene NW, Graves DJ. (2004) Isolation and characterization of chalconepolymers from cinnamon with insulin like biological activity. Journal of Agricultural and Food Chemistry, 14(52), 65-70.

[6] Khan A., Safdar M, Khattak MMAK, Siddique M. (2004). Effect of various doses of cinnamon on blood glucose in diabetic individuals. Pakitan Journal Nutrition, 3, 268-272.

[7] Weyer C, Bogardus C, Mott DM, Pratley RE. (1999). The natural history of insulin secretory dysfunction and insulin resistance in the pathogenesis of type 2 diabetes mellitus. Journal of Clinical Investigation, 104, 787-794.

[8] Comuzzie AG, Funahashi T, Sonnenberg G, Martin LJ, Jacob HJ, Black AE, Maas D, Takahashi M, Kihar S, Tanaka S, Matsuzawa Y, Blangero J, Cohen D, Kissebah A. (2001). The genetic basis of plasma variation in adiponectin, a global endophenotype for obesity and the metabolic syndrome. The Journal of Clinical Endocrinology and Metabolism, 86, 4321-4325.

[9] Yang WS, Lee WJ, Funahashi T, Tanaka S, Matsuzawa Y, Chao CL, Chen CL, Tai TY, Chuang LM. (2001). Weight reduction increases plasma levels of an adipose-derived anti-inflammatory protein, adiponectin. The Journal of Clinical Endocrinology and Metabolism, 86, 3815-3819.

[10] WHO (2006), Definition and diagnosis of diabetes mellitus and intermediate hyperglycaemia: A report of WHO/ IDF consultation, Geneva WHO.

[11] Khan A, Safdar M, Khattak MMAK, Khattak KN, Anderson RA (2003). Cinnamon improves glucose and lipids of people with type 2 diabetes. Diabetic Care, 26, 3215-3218.

[12] American Diabetes Association (ADA) (2003). "Diabetes Symptoms." American College of Endocrinology.Position statement on inpatient diabetes and metabolic control. Endocrinology Practice, 10, 77.

[13] Mahmood T, Khattak MMAK, Mohd-Shukri NA, Azam SA, Aimi N. (2014). The Effect of Mixed Herbs on Blood Glucose and Lipid Profile in Case Controlled Type 2 Diabetes Mellitus (T2DM). The 2nd USM International Nursing Conference (2nd USM INCON 2014).

[14] Mahmood T, Khattak MMAK, Mohd-Shukri NA. Azam SA, Shamsuddin, NA (2014). Effect Of Mixed Spices On Hyperglycemia \& Lipid Profile In Case Controlled Type 2 Diabetes Mellitus (T2DM): A preliminary report. In 3rd Annual Global Healthcare Conference (GHC 2014), No. 3. (23-24 June 2014).

[15] Mahmood T, Khattak MMAK, Mohd-Shukri NA, Zulkifli HH, Azam SA. (2014). Consumption of Mixed Spices and Herbs and General Well-Being Observed in Case Controlled Type 2 Diabetes Mellitus (T2DM). 2014 International Conference on Food Security and Nutrition. CBEE vol. 67 (2014) (C) (2014) IACSIT Press, Singapore. 